\title{
Protokoll der Mitgliederversammlung vom 20. September 2019 in Münster
}

\section{Beginn 16:45 Uhr, Ende 18:30 Uhr, Teilnehmer: 78}

Der Präsident, Prof. Markus Stücker begrüßt die Teilnehmer und stellt fest, dass die Versammlung mit Veröffentlichung der Tagesordnung im Heft 4/2019 der Zeitschrift Phlebologie satzungsgemäß einberufen wurde.

Die Teilnehmer der Mitgliederversammlung erheben sich für eine Schweigeminute zu Ehren der seit der letzten Mitgliederversammlung verstorbenen Mitglieder.
TOP 1: Genehmigung der Tagesordnung

Die Tagesordnung wird einstimmig angenommen.

TOP 2: Genehmigung des Protokolls der Mitgliederversammlung vom 28.9.2018

(veröffentlicht in Phlebologie 4/2018)
Das Protokoll wird einstimmig genehmigt.

\section{TOP 3: Bericht des}

\section{Präsidenten}

Prof. Stücker begrüßt die Teilnehmer und bedankt sich bei allen aktiven Mitgliedern in der Deutschen Gesellschaft für Phlebologie. Eine wissenschaftliche Fachgesellschaft könnte ohne deren Engagement nicht bestehen. Darüber hinaus berichtet er über die Entwicklungen der letzten Monate. 


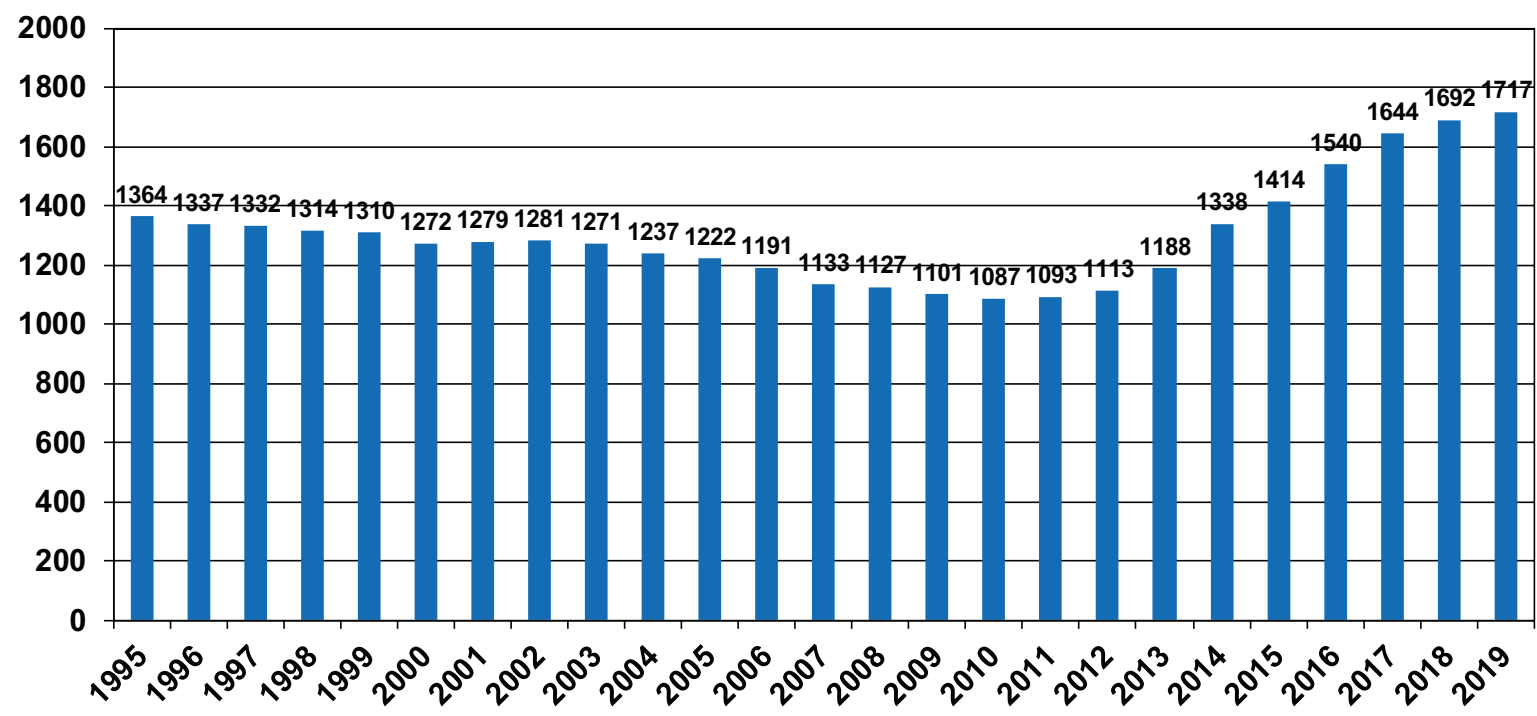

-Abb. 1 Mitgliederentwicklung

Insgesamt ist die Mitgliederentwicklung der DGP nach wie vor erfreulich, wobei der Anstieg sich im Augenblick eher abflacht. Die DGP ist nach der DGG die zweitgrößte Gefäßgesellschaft.

Bei den Facharztgruppen sind nach wie vor die Dermatologen und die Gruppe der Chirurgen/Gefäßchirurgen, gefolgt von den Allgemeinmedizinern als größte Gruppe auszumachen. Es wird vorgeschlagen, sich in diesen Bereichen verstärkt um Neumitglieder zu bemühen. Hier bieten sich Aktivitäten im Bereich „Phlebologie für den Hausarzt“ und auch Kurse mit dem Titel „Phlebologisches Basiswissen - was ich als Chirurg/Gefäßchirurg brauche“ im Rahmen der jeweiligen Fachtagungen oder bei lokalen kleineren Veranstaltungen (z. B. DWFA) an.

Die Zahl der außerordentlichen und fördernden Mitglieder ist konstant auf einem eher niedrigen Niveau. Hier scheint v. a. der Bereich der außerordentlichen Mitglieder aus den Bereichen Pflege und Sanitätshaus ausbaufähig. Für die Zukunft sollte gerade das Update Wundmanagement verstärkt beworben werden.

Folgende Wissenschaftspreise werden in diesem Jahr in Kooperation mit der DGP verliehen:

Bauerfeind Doktorandenpreis (Jury bestehend aus E. Rabe, M. Stücker, Vertreter Bauerfeind) ( $\triangleright$ Abb. 2)
Carolin Mitschang, Universitätshautklinik Münster: Die Lebensqualität von Patientinnen und Patienten mit diagnostizierter Stammveneninsuffizienz vor und nach Durchführung einer Radiofrequenzablation (betreut durch Prof. Görge)

Irene (Joana) Döll, Universitätsmedizin Greifswald - Dermatologie: Evaluation von Akzeptanz und Nutzerzufriedenheit nach teledermatologischer Behandlung in der Zentralen Notaufnahme der Universität Greifswald mit Hilfe des Teledermatologiesystems Mobil Skin ${ }^{\circledR}$ (betreut durch Prof. jünger.)

Juzo Forschungspreis Phlebologie (Jury bestehend aus Kongresspräsident, E. Valesky, Vertreter von Juzo) ( $>$ Abb. $\mathbf{3}$ )

Jana Ziob, Bonn

SIGVARIS Förderpreis Phlebologie (Preisträger wird von der Firma Sigvaris ausgewählt) (>Abb. 4)

Dr. med. Christine Fink, Heidelberg (3500,- $€$ für phlebologische Kongresse und Fortbildungen mit paralleler Berichterstattung)

\section{SIGVARIS Studentenpreis Phlebologie} (Preisträger werden von der Firma Sigvaris ausgewählt) ( $\triangleright$ Abb. 4)

Hanna Brockhoff, Bochum im 8. Semester (1000,- € für phlebologische Fortbildungsveranstaltungen der Deutschen Gesellschaft mit paralleler Berichterstattung)

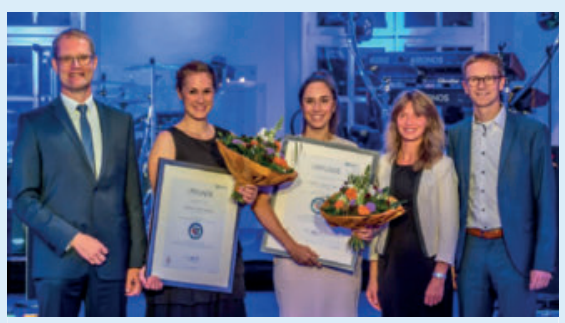

Abb. 2 Prof. Stücker, Carolin Mitschang, Irene Döll, Dr. Mark (Bauerfeind), Prof. Görge

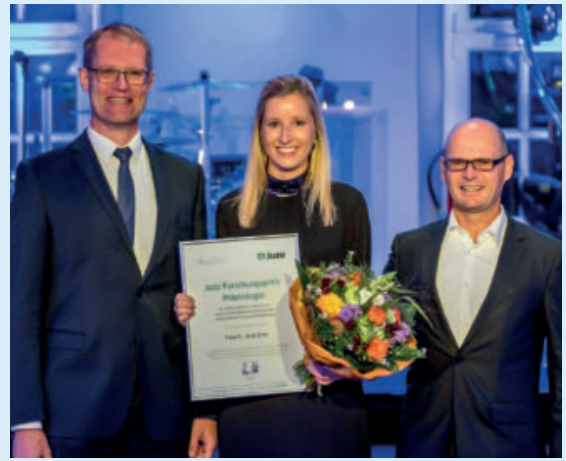

Abb. 3 Prof. Stücker, Dr. J. Ziob, U. Frey (Firma Juzo)

Kreussler Travel Awards (aus den eingereichten Abstracts d. Kreussler) (\Abb. 5) Thea Westphal, Greifswald: „Messung der gesundheitsbezogenen Lebensqualität bei CVI (TLQ-CVI): Einfluss eines MKS“

Olivia Danneil, St. Josef-Hospital, Bochum: „Tiefe Muskelvenenthrombose nach Sklerosierung eines Seitenastes unter laufender Therapie mit Tamoxifen“ 

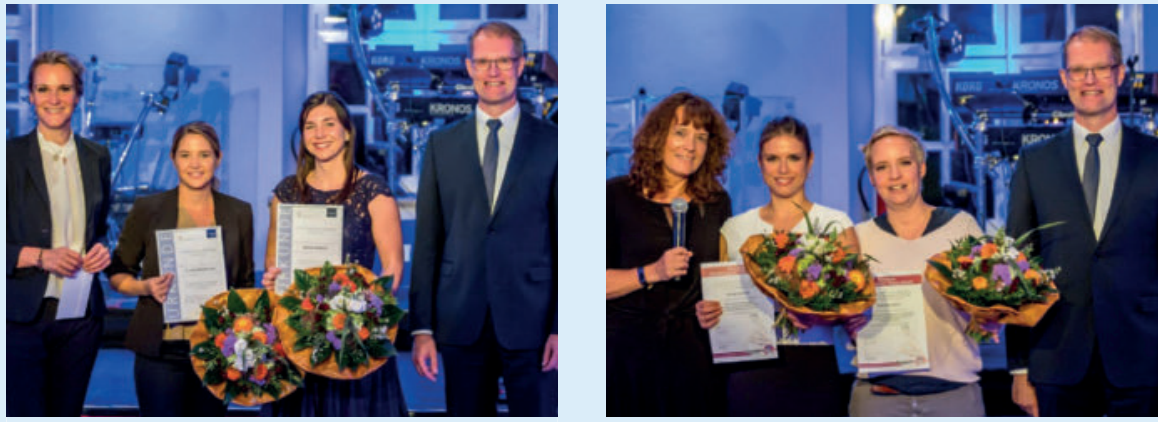

Abb. 4 C. Töpfer (Fa. Sigvaris), Dr. C. Fink, H. Brockhoff, Prof. Stücker

Abb. 5 Dr. P. Gliem (Fa. Kreussler), Dr. O. Danneil, Dr. S. Lutze (in Vertretung)

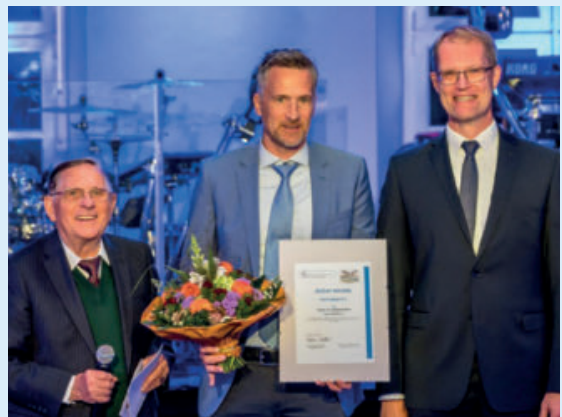

Abb. 6 Prof. Varady, Dr. Böhm, Prof. Stücker

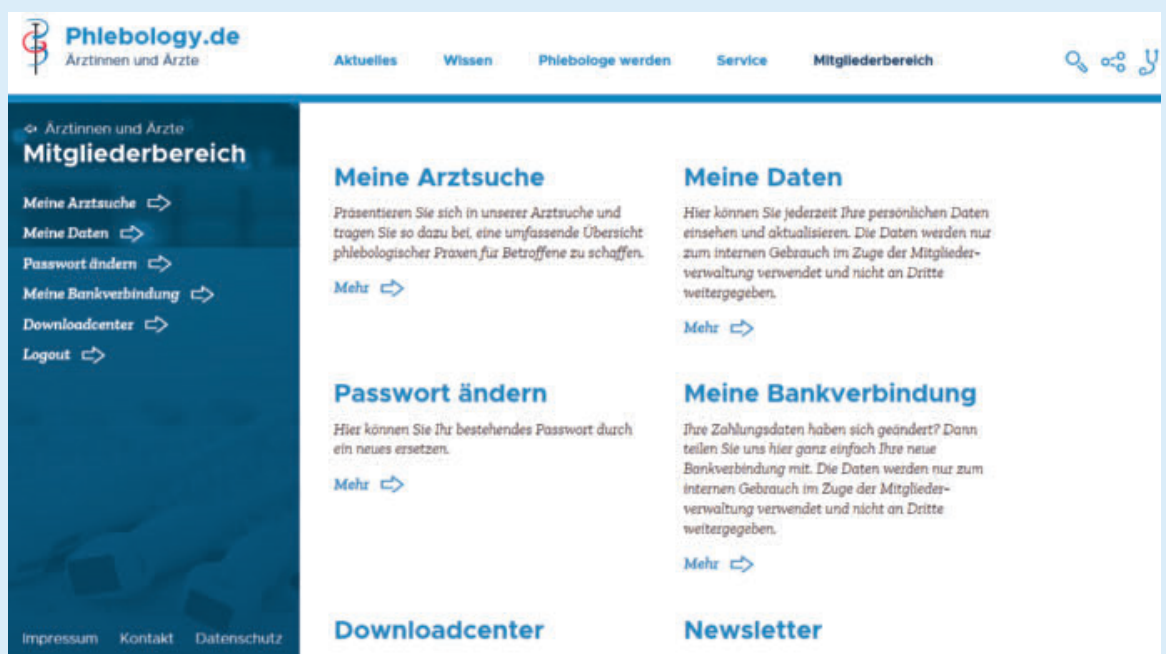

Varady Vortragspreis (Jury besteht aus Mitgliedern der VOP AG - D. Mühlberger, A. Mumme, K. Hartmann) Preisträger wird aus den thematisch passenden Vorträgen ausgewählt ( Abb. 6)

Medi Reisestipendien In diesem Jahr wurden 15 Preise vergeben, die Auswahl und Bewerbung erfolgt direkt über die Firma Medi.

Prof. Stücker bittet hier, diese Preise aktiv zu bewerben! Machen Sie Werbung für die Wissenschaftspreise und sprechen Sie Ihre Mitarbeiter und Mitarbeiterinnen an!

Die Teilnehmerzahl der DGP-Jahrestagungen hat sich auf einem gleichbleibenden Niveau um die 1.000 Teilnehmer eingependelt. Dies ist einerseits erfreulich, andererseits bedeutet es allerdings auch eine Reduzierung gegenüber 2015 um 15\%. Alternative Ideen wurden in einen Fragebogen integriert, der an der Anmeldung ausliegt. Dieser ist dem Protokoll angehängt. Prof. Stücker bittet darum, diesen ausgefüllt an der Anmeldung abzugeben.
Fortbildungsakademie Die Fortbildungsmaßnahmen der DGP werden gut angenommen. Mittlerweile haben 166 Ärzte das Fortbildungszertifikat Phlebologie erworben. Durch die Kopplung an das gemeinsam von der DGP und dem Berufsverband herausgegebene Qualitätssiegel VKZ plus hat sich die Zahl im letzten Jahr deutlich gesteigert. Insgesamt haben 185 Praxen das VKZ-Siegel, 22 Praxen tragen das VKZ plus Siegel.

Die Frage der Weiterentwicklung der Zusatzbezeichnung Phlebologie (sowohl berufsbegleitend als auch in ihrer bisherigen Form) ist nach wie vor nicht geklärt. Neuer Ansprechpartner bei der Ärztekamme ist Herr Herrmann, Mitglied der Ärztekammer desärztekammer, wie die berufsbegleitende Weiterbildung in die Praxis umgesetzt werden soll und wie der fachlich empfohlene Weiterbildungsplan umgesetzt werden soll, sollen laut BÄK im Herbst formuliert werden, auch anhand des von uns bereits mehrfach eingereichten Entwurfs für einen Schleswig-Holstein. Die Vorgaben der Bun- fachlich empfohlenen Weiterbildungsplan. Zunächst möchte die DGP auf diese Vorgaben Einfluss nehmen, erst danach auf die verschiedenen Landesärztekammern. Jeder sollte versuchen, Kontakt zu den Ausschussmitgliedern der ständigen Kommission Weiterbildung zu suchen, um die Vorgaben der BÄK mitgestalten zu können.

Der Weltthrombosetag findet wie in diesem Jahr am 11.10.2019 unter dem Motto „Alte Zöpfe abschneiden: Lungenembolie ambulant behandeln!“ Das Thema wird wieder in Berlin mit namhaften Wissenschaftlern diskutiert. Prof. Bauersachs und Dr. Jutta Schimmelpfennig vertreten die DGP.

Darüber hinaus wird noch einmal auf die Informationsmaterialien des Aktionsbündnisses Thrombose hingewiesen, die kostenlos zur Ausgabe an Patienten angefordert werden können.

Website Dr. Bruning berichtet über die Neugestaltung und inhaltliche Überarbeitung der Website, die 2018/2019 erfolgt ist.

Insgesamt zeigen die Zugriffszahlen, dass die Website gut angenommen wird. Die Zugriffe erfolgen im wesentlich über mobile Endgeräte wie Smartphone und Tablet. Die Zugriffe erfolgen im Wesentlichen über Suchmaschinen, wobei die verwendeten Suchbegriffe eher „laienorientiert“ sind.

Klarer Spitzenreiter bei den Suchbegriffen ist die „Arztsuche“, die sowohl von Patienten als auch von Ärzten genutzt wird. An dieser Stelle noch einmal der Hinweis, dass sich bitte alle Mitglieder auf der Website registrieren sollten. Dies sollte in zwei Schritten erfolgen: 

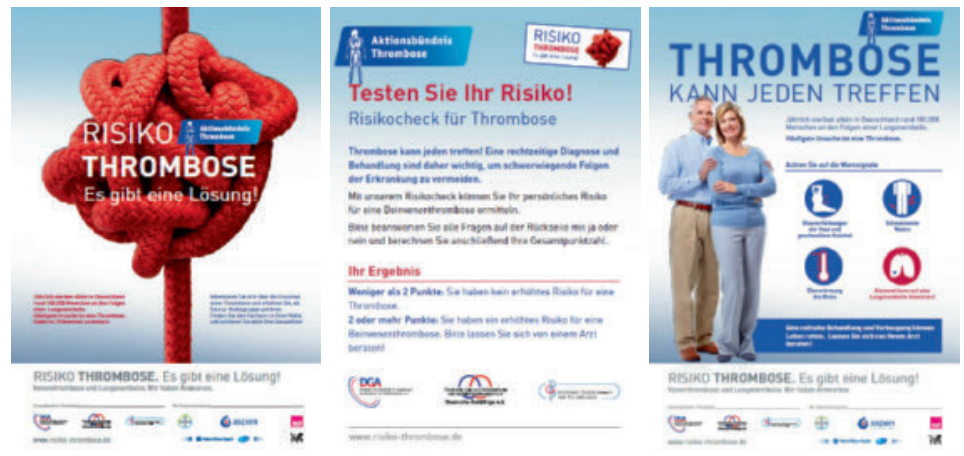

1. Meine Daten - hier verwalten Sie Ihre Daten, die nicht der Veröffentlichung dienen

2. Meine Arztsuche - hier legen Sie Ihr Arztprofil für die Arztsuche fest.

\section{TOP 4: Bericht der Generalsekretärin}

Liposuktion Frau Dr. Mendoza berichtet über die Aktivitäten sowohl von GBA als auch von DGP zum Thema Liposuktion. Nach Bekanntwerden der Pläne von GBA/ Gesundheitsministerium, eine Änderung der Richtlinie zur Qualitätssicherung der Liposuktion bei Lipödem im Stadium III sowie zu den Untersuchungs- und Behandlungsmethoden im Krankenhaus und bei der vertragsärztlichen Versorgung anzustreben, wurde von der DGP eine Taskforce Lipödem/ Liposuktion der Deutschen Gesellschaft für Phlebologie gemeinsam mit der Deutschen Gesellschaft für Lymphologie gegründet. Teilnehmer hier waren: Dr. med. Gabriele Faerber, Dr. med. Tobias Hirsch, Dr. med. Karsten Hartmann, Dr. med. Tobias Bertsch, Prof. Dr. med. Markus Stücker. Es wurde eine Stellungnahme von DGP und DGL abgegeben.

Das GBA-Papier kommt zum Ergebnis, dass der Nutzen der Liposuktion im Stadium III des Lipödems als hinreichend belegt und die medizinische Notwendigkeit als gegeben anzusehen ist, wenn dieses Stadium trotz des Einsatzes konservativer Maßnahmen entstanden ist, weil dann keine therapeutischen Alternativen zur Liposuktion mehr verfügbar seien. Die Studienlage nicht „hinreichend belegt“ und vorhandene Studien seien monozentrische Studien, die von liposuktionsdurchführenden Kollegen selbst durchgeführt wurden. Multizentrische Stu- dien bzw. Studien aus interdisziplinären Arbeitsgruppen operativ und nicht operativ tätiger Ärzte existieren nicht.

Frau Dr. Mendoza berichtet über die Anhörung vor dem GBA, an dem sie gemeinsam mit Frau Dr. Miller für die DGP teilgenommen hat. Zur Diskussion steht die Definition einen BMI<32 einem Lipödem Stadium III zuzuordnen und dann Operationen durchzuführen. Die Entscheidung beim GBA wurde am 19.09.2019 getroffen. Ergebnis: Der gemeinsame Bundesausschuss (G-BA) hat die Kostenübernahme für die Liposuktion ausschließlich im Stadium III bis zu einem BMI von $40 \mathrm{~kg} / \mathrm{m}^{2}$ unter Auflagen genehmigt. Da es sich beim Lipödem im Stadium III ohne begleitende Adipositas um ein relativ seltenes Krankheitsbild handelt, empfiehlt die Deutsche Gesellschaft für Phlebologie die zusätzliche Dokumentation der WHtR als Entscheidungsgrundlage. Leider ist die vom G-BA geplante Studie zur Wirksamkeit der Liposuktion bei Lipödem noch nicht abgeschlossen, daher wissen wir heute nicht, ob diese Maßnahme für die Patientinnen sinnvoll ist. Hierzu gibt die DGP zusammen mit der DGL und GDL eine Pressemitteilung heraus.

EVF-Tagung Frau Dr. Mendoza berichtet über die EVF-Tagung in Zürich. Die EVF-Tagung ist als einzügige Veranstaltung konzipiert mit streng nach Abstract ausgewählten wissenschaftlichen Sitzungen (aus 400 Abstracts werden 40 Vorträge selektiert), LehrSitzungen (Organisiert vom Gastgeber), Postersitzungen (eine am Tag) und eine AVFKoop.-Sitzung.

Da es keine Möglichkeit einer Back to Back Veranstaltung mit der DGP Jahrestagung gibt, wurde entschieden die EVF-Tagung im
Juni in Berlin und die DGP -Tagung im September in Hannover zu veranstalten. Von den möglichen Synergien für die Organisation (v. a. Abstract- und Industrieakquise) profitieren sicher beide Veranstaltungen. Frau Dr. Mendoza bittet die deutschen universitären Einrichtungen, jetzt schon Studien aufzulegen, die dann am EVF präsentiert werden könnten, damit auch eine deutsche Beteiligung unter den Vorträgen zu erwarten ist.

Satzungsänderung Im Rahmen des UIPChapter Meetings wurde dort in der Vorstandsitzung eine Satzungsänderung angestrebt. Aufgrund der Nichteinhaltung von Fristen musste die Entscheidung auf Buenos Aires 2020 verschoben werden. Neuer UIP Vorsitzender ist Kurosh Parsi, das nächste UIP-Meeting findet 2023 in Miami statt.

International Ambassador Project Frau Dr. Mendoza berichtet über ein „International Ambassador Project“, das dazu dienen soll, Länder und deren Ärzte mit unzureichender phlebologischer Versorgung phlebologisch fortzubilden und es ihnen zu ermöglichen, sich als Fachgesellschaft zu organisieren. Die Idee ist es, durch die Vernetzung mit ausgewanderten Landsleuten, die in Deutschland leben und arbeiten, die Ursprungs-Gesellschaften in ihren Ländern zu unterstützen. In Im Libanon ist das erste Ambassador - Projekt mit Australien angelaufen. Eine Vernetzung mit Deutschland würde gern angestrebt werden. Hier könnten libanesische Ärzte in Deutschland angesprochen werden, sich dort einzubringen. Frau Dr. Mendoza würde dies Projekt gern für Deutschland koordinieren. Darüber hinaus wäre es im Rahmen der Jahrestagung möglich, eine „Ambassador“ - Sitzung einzurichten oder es könnten englische Masterclasses angeboten werden zu verschiedenen Themen, wie Ultraschall, endoluminale Therapien, Schaumverödung oder Ulkustherapie, die dann auch für alle Teilnehmer offen stünden.

\section{TOP 5: Bericht des \\ Schatzmeisters}

Die finanzielle Situation der DGP ist solide.

2018 hat die DGP Einnahmen aus Teilnehmergebühren, Pachterträgen der Jahres- 
tagung und aus der Fortbildungsakademie in Höhe von $€ 216565$,78 erwirtschaftet. Demgegenüber standen Ausgaben in Höhe von 135 556,47, was einem Gewinn von 81 009,41 für das Jahr 2018 entspricht.

Das momentane Guthaben der DGP beläuft sich auf rund $€ 200000,00$.

Dr. Hartmann regt noch einmal an, Forschungsförderungsanträge zu stellen. Das Einreichungsverfahren wird auf der DGPWebsite erläutert.

\section{TOP 6: Bericht der \\ Kassenprüfer - Entlastung des Vorstandes}

Herr Dr. Neff berichtet über die Kassenprüfung und bescheinigt ordentliche Rechnungsführung. Die beantragte Entlastung des Vorstandes erfolgt einstimmig bei 6 Enthaltungen.

\section{TOP 7: Wahlen}

Frau Dr. Reich-Schupke kandidiert aus gesundheitlichen Gründen nicht mehr für den wissenschaftlichen Beirat, so dass ein Platz frei wird.

Prof. Stücker dankt für ihr Engagement in der Vergangenheit!

Mögliche Kandidaten:

- Dr. Andreas Bayer, Lübeck

- Dr. Hans Bayer, Freiburg

- Dr. Christine Fink, Heidelberg

- Dr. Houman Jalaie, Aachen

- Dr. Stine Lutze, Greifswald

- Dr. Johann Ragg, Berlin

- Dr. Jasmin Woitalla-Bruning, Hamburg

Gewählt wurde: Dr. Jasmin Woitalla-Bruning, Hamburg (die Kandidatin nimmt die Wahl an).

Als Kassenprüfer werden benannt: Dr. Heisterkamp, Gescher und Dr. Neff, Eitorf.

\section{TOP 8: Bericht der Kongresspräsidenten}

Münster Prof. Görge bedankt sich bei dem aktiven wissenschaftlichen Beirat. Insgesamt sind 43 Industriesponsoren dabei. Al- lerdings muss man bemerken, dass sich die Gesamtinvestitionen, v. a. der Hauptsponsoren reduziert haben. Einige Sponsoren wir OFA und BSN haben sich komplett zurückgezogen.

Prof. Stücker dankt dem Kongresspräsidenten noch einmal ausdrücklich für die Organisation der Tagung.

Leipzig Die Vorbereitungen für die Jahrestagung in Leipzig laufen „nach Plan“. Die bewährten Kernelemente Crash-Kurs Phlebologie, Update Phlebologie, Update Wundmanagement und Weiterbildungsforum werden beibehalten. Ein weiterer Schwerpunkt wird auf der Förderung der Internationalität der DGP-Jahrestagung liegen. Es soll einen englischsprachigen Strang geben, in dem Simultanübersetzungen angeboten werden. Besonders die osteuropäischen Nachbarländer sollen mit einem speziellen englischsprachigen Flyer angesprochen werden.

Zur Information die Termine der Jahrestagungen der nächsten Jahre:

- 2020: 02.-05.09.2020 (Tobias Hirsch, Leipzig)

- 2021: 7.-11.9.2021 (Knuth Rass/

Houman Jalaie, Aachen)

- 2022: EVF - 22.06.-25.06.2022

(Erika Mendoza, Berlin)

- 2022: DGP - 28.09.-30.09.2022

(Erika Mendoza, Hannover)

- 2023: 13.-16.09.2023 (Bruno Geier, Meike Finkenrath, Duisburg)

\section{Top 9: Forschungsbericht}

Die Zahl der Veröffentlichungen von Originalarbeiten von DGP-Mitgliedern in Pubmed gelisteten Zeitschriften liegt bei 144, 33 Veröffentlichungen kamen aus der Phlebologie.

Das Forschungsantragsprozedere wurde leicht verändert, nachzulesen auf der Website. Die Mitglieder werden aufgefordert, Forschungsanträge einzureichen.

\section{TOP 10: Bericht aus dem Berufsverband}

Der BVP ist weiter aktiv in der EBM Reform und GOÄ Entwicklung tätig.
In Zusammenarbeit mit der AG Endo ist das Gütesiegel VKZplus verabschiedet und aktiviert worden und findet allgemeine Zustimmung. Die weiteren möglichen Sektionen wie die AG Sklero und AG VOP sind angesprochen und können mitmachen.

Eine Abrechnungsempfehlung zur Liposuktion wurde in Zusammenarbeit mit dem BVP entwickelt und vorgestellt.

Der BVP hat auch weiterhin medizinischjuristisch heikle Fragen zur Abklärung gebracht bzw. bearbeitet:

- so z. B. die Frage der off-label-Behandlung mit der Tumeszenzanästhesie,

- arbeitsrechtliche und vertragsrechtliche Fragen zur „begleitenden Weiterbildung im Rahmen der neuen WBO“.

Der BVP berät die KBV bei der Einführung einer EBM Abrechnungsziffer zur Liposuktion und hat bei BGM Spahn heftigsten Protest dagegen eingelegt, dass ohne GBA Entscheidung zur Methode und Indikationsstellung die GKV Abrechnung ermöglicht werden soll, ohne dass „neues Geld“ dafür in die Abrechnung kommt.

\section{TOP 11: Leitlinien}

In diesem Jahr sind folgende Leitlinien bei der AWMF veröffentlicht worden:

Leitlinie medizinische Kompressionstherapie der Extremitäten mit Medizinischem Kompressionsstrumpf (MKS), Phlebologischem Kompressionsstrumpf (MKS), Phlebologischen Kompressionsverband (PKV) und Medizinischen adaptiven Kompressionssystemen (MAK)

Rabe E, Földi E, Gerlach H, Jünger M, Lulay G, Miller A, Protz K, Reich-Schupke S, Schwarz T, Stücker M, Valesky E, Pannier F

Leitlinie Sklerosierungsbehandlung der Varikose

Rabe E, Breu FX, Flessenkämper I, Gerlach H, Guggenbichler S, Kahle B, Murena R, ReichSchupke S, Schwarz T, Stücker M, Valesky E, Werth S, Pannier F

Leitlinie Diagnostik und Therapie der Varikose

Pannier F, Noppeney T, Alm J, Breu FX, Bruning G, Flessenkämper I, Gerlach H, Hartmann K, Kahle B, Kluess H, Mendoza E, Mühl- 
berger D, Mumme A, Nüllen H, RassK, ReichSchupke S, Stenger D, Stücker M, Schmedt CG, Schwarz T, Tesmann J, Teßarek J, Werth $\mathrm{S}$, Valesky $\mathrm{E}$

Prof. Stücker dankt den Leitlinienkoordinatoren und allen Beteiligten für Ihr Engagement bei der Erstellung der Leitlinie!

\section{TOP 12: Fortbildungs- akademie}

Insgesamt gibt es im Augenblick 166 Inhaber des phlebologischen Fortbildungszertifikats, Verlängerungen laufen unproblematisch.

Im Jahr 2019 haben DGP und Berufsverband der Phlebologen das VKZplus-Qualitätssiegel initiiert. Der Erwerb dieses Siegels ist an das phlebologische Fortbildungszertifikat gekoppelt. Das Ziel des VKZplus ist es ein erweitertes Qualitätssicherungssystem für die phlebologische Fachversorgung zu schaffen, um die Qualität in der invasiven Therapie der Versorgung von Venenerkrankungen zu erhöhen und einen ausreichenden Ausbildungsnachweises für die spezialisierte integrierte Arbeitsmethode, also aktuell für die endovenösen thermalen Ablationsverfahren (EVTA) sicher zu stellen.

\section{TOP 13: Bericht aus der Zeitschrift Phlebologie}

Bei Veröffentlichungen in Phlebology oder anderen englischsprachigen internationalen Zeitschriften weisen die Schriftleiter der Phlebologie darauf hin, dass für Einreichungen in der Phlebologie gute Chancen zur Veröffentlichung bestehen.
Ebenso wird noch einmal darauf hingewiesen, möglichst viel aus der Phlebologie bei internationalen Publikationen zu zitieren.

Die Manuskriptakquirierung läuft zufriedenstellend, ist aber ausbaufähig.

Es werden regelmäßig Themenhefte herausgegeben. Aktuell wird an einer Ausgabe zur „Kosmetischen Phlebologie“ gearbeitet.

Top 14: Berichte aus den Kommissionen/ Arbeitsgemeinschaften

In der DGP sind im Augenblick folgende Arbeitsgemeinschaften aktiv:

- Sklero-AG (Breu/Guggenbichler/Kahle/ Murena)

- Es wird über eine Studie zur Sklerosierung der Seitenastvarikose nachgedacht.

- Endo AG (Hartmann/Pannier)

- Es wird über einen Studieninitiierung nachgedacht.

- OUT AG (Hermanns)

- VOPAG (Stenger/Mumme)

- Die Ergänzung/Fortführung der LaVaCro Studie ist geplant.

- AG Lymphologie (Schwahn-Schreiber)

- Organisation von wissenschaftlicher Sitzung bei Jahrestagung

- Weiterbildung von Kollegen und Lymphtherapeuten in Zusammenarbeit mit LymphologicAG Venöse Malformationen (Berlien)

- AG Hämodynamik (Mendoza)

- Arbeit an einem Konsens zur Dokumentation
- AG Thrombose

- funktioniert als interaktives Chatpodium, auf dem man sich austauschen kann und über das Expertenmeinungen angefragt werden können.

- Beantwortung von spezifischen Anfragen zur VTE von Kollegen*innen und der Fachpresse

- Versteht sich als Netzwerk, welches das Wissen um die Thromboembolie, auf hohem und aktuellem Niveau in die Phlebologie einbringen kann.

- AG Arbeitsmedizin (Gallenkemper)

- AG Junge Phlebologen (Mühlberger)

- AG Hilfsmittelversorgung (Valesky)

- AG Pflege (Hampel-Kalthoff)

- AG Phlebologieassistenten (Murena)

- Insgesamt haben 149 Teilnehmer*innen die Ausbildung abgeschlossen

- Treffen der Phlebologieassistenten im Rahmen der Jahrestagung

- Insgesamt nur 7 abgebrochene Ausbildungen

Gez.

Prof. Dr. med. Markus Stücker

Präsident

Dr. med. Erika Mendoza

Generalsekretärin

IMPRESSUM

Verantwortlich für Mitteilungen der DGP:

Dr. med. Erika Mendoza 